Grâce à la brillance des

sources synchrotron de

troisième génération,

on obtient des faisceaux

de rayons $X$ cohérents

d'intensité supérieure à

$10^{9}$ photons par seconde.

Ces faisceaux permettent

des expériences de diffraction

qui révèlent les franges

d'interférence ou les tavelures,

non visibles dans

des expériences classiques.

Cet article donne quelques

exemples d'études en

diffraction cohérente :

reconstruction de déforma-

tions dans des nanocristaux,

mise en évidence de défauts

topologiques, et ptychogra-

phie. Des expériences de

diffraction cohérente sont

notamment réalisées sur

la ligne CRISTAL à SOLEIL, ainsi qu'à I'ESRF.

\section{La diffraction cohérente des rayons $X$}

Sylvain Ravy (sylvain.ravy@synchrotron-soleil.fr)

Synchrotron SOLEIL, L'Orme des Merisiers, Saint-Aubin, BP 48, 91192 Gif-sur-Yvette Cedex
La diffraction des rayons $\mathrm{X}$ est la méthode reine pour obtenir des informations sur la structure atomique de la matière. Parce que les rayons $\mathrm{X}$ ont des longueurs d'onde $\lambda$ de l'ordre de grandeur des distances interatomiques, des interférences sont possibles entre les faisceaux diffusés par les atomes. Ces interférences donnent naissance à des diagrammes de diffraction composés de taches, les taches de Bragg, dont la position et l'intensité sont reliées à la position relative des atomes dans le cristal. Étonnamment, un faisceau de rayons $\mathrm{X}$ n'a pas besoin d'être très cohérent pour donner telles taches : il suffit qu'il le soit sur quelques distances interatomiques..

Cependant, le manque de cohérence des rayons $\mathrm{X}$ se fait sentir dans certaines expériences. Par exemple, le diagramme de diffraction d'un petit cristal de taille finie $a$ est composé de taches de Bragg flanquées d'une série de franges séparées angulairement $\mathrm{de} \sim \lambda / \mathrm{a}$ (figs. $2 \mathrm{a}-\mathrm{b}-\mathrm{c}$ ). Malheureusement, ces franges d'interférences, qui contiennent également des informations sur ce cristal, ne sont jamais observées en diffraction classique, car les sources de rayons $\mathrm{X}$ ne sont pas assez cohérentes.

Dans ce court article, nous montrons comment l'utilisation de la cohérence des sources de rayons $\mathrm{X}$ les plus récentes permet d'obtenir de nouvelles informations sur la structure des solides.

\section{Cohérence des sources de rayons $X$}

Qu'elle consiste en un tube de laboratoire où en un onduleur d'un centre de rayonnement synchrotron, une source de rayons $\mathrm{X}$ est étendue et incohérente : chaque point de cette source de taille $S$ émet un faisceau indépendamment des autres. Cependant, grâce à la petite taille des sources synchrotron et à la grande longueur des lignes de lumière, ces faisceaux peuvent acquérir par simple propagation une cohérence transverse suffisante pour être utilisables.

La diffraction par des trous de Young permet de définir simplement la notion de cohérence transverse (fig. 1). Les rayons issus du centre d'une source située à une distance $D$ de deux trous de Young séparés de $a$, donnent sur un détecteur éloigné une série de franges d'interférence, angulairement séparées de $\lambda / a$. Le point situé en $S / 2$ à l'extrémité de la source donne la même série de franges, décalée de l'angle $S / 2 D$. Si cet angle est égal à $\lambda / 2 a$, les franges claires issues du centre de la source et les franges sombres issues de son extrémité se superposeront et seront donc invisibles. La condition de visibilité des franges s'écrit alors :

$$
\lambda D / S \equiv L_{T} \geq a \text {. }
$$

Ainsi définie, $L_{T}$ est la longueur de cohérence transverse du faisceau : c'est la longueur sur laquelle on peut considérer

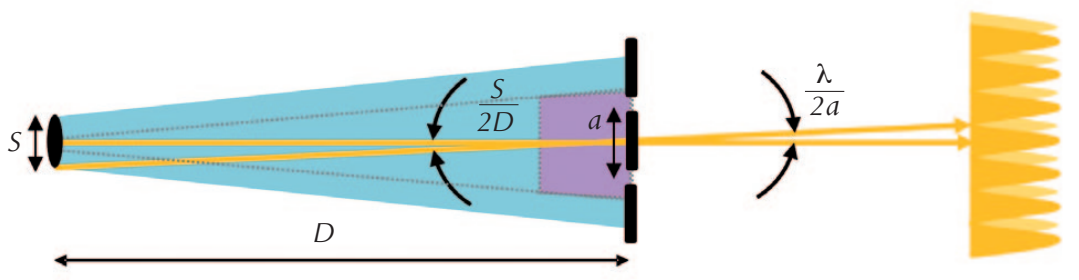

1. Représentation schématique de la diffraction par deux trous de Young séparés de $a$. Le faisceau est représenté en bleu et sa partie cohérente, limitée par $L_{T}$ et $L_{L}$, en mauve. Les franges de Young issues du centre de la source sont en orange, celles issues de son extrémité sont en jaune. 

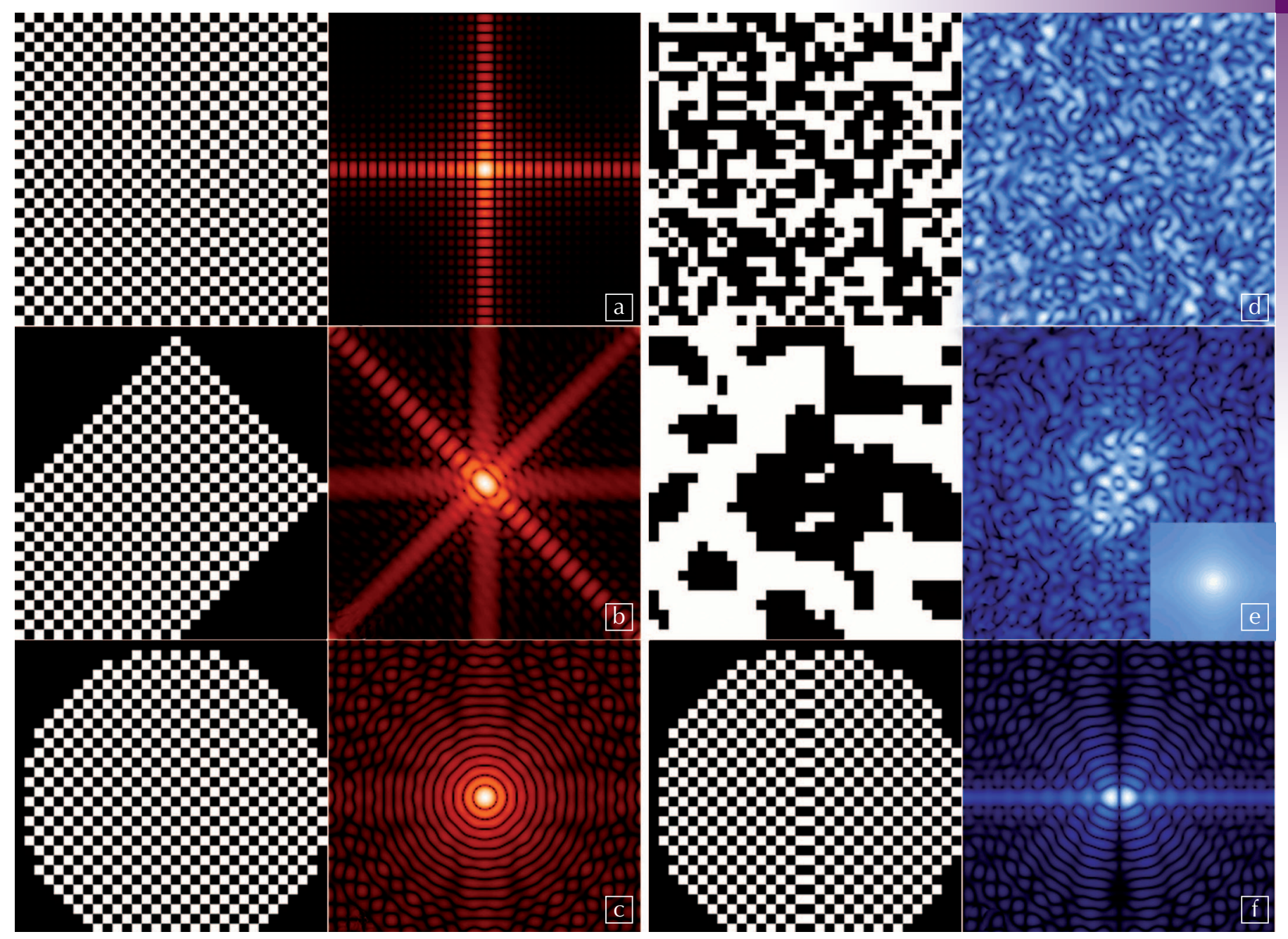

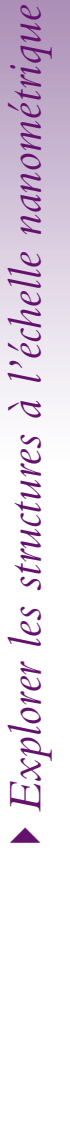

2. Exemples de diagrammes de diffraction cohérente (en couleurs) de différents objets (en noir et blanc), obtenus par simulation. L'échelle des couleurs est logarithmique.

Les figures de gauche $(a, b, c)$ montrent de petits cristaux ordonnés de formes différentes et de tailles finies. Les franges d'interférences autour du maximum principal (la tache de Bragg) sont caractéristiques de la taille du cristal.

Les figures de droite (d, e, f) montrent des exemples de désordre ou de défauts. En d), les atomes, représentés en blanc, sont disposés aléatoirement sur les sites du cristal. En e) ces atomes tendent à s'agréger en domaines ; la diffusion diffuse, montrée en encart en e), est résolue en tavelures ou speckles. L'exemple f) montre une paroi de domaine : la tache de Bragg observée dans le cas c) est maintenant dédoublée.

Dans les six cas présentés, la largeur des franges d'interférence ou des tavelures est la même : l'inverse de la taille du carré.

que l'onde incidente est plane. Si l'objet diffractant est plus petit que $L_{T}$, les franges d'interférence deviennent visibles. Sur la ligne CRISTAL de SOLEIL par exemple, à une distance $D=40 \mathrm{~m}$ et avec une longueur d'onde $\lambda=1,8 \AA(7 \mathrm{keV})$, cette longueur vaut $L_{T V} \cong 350 \mu \mathrm{m}$ verticalement et $L_{T H} \cong 10 \mu \mathrm{m}$ horizontalement. Le faisceau de lumière de fréquence $v$ est également caractérisé par sa largeur spectrale $\Delta v$. Il possède donc un temps de cohérence de l'ordre de $1 / \Delta v=c \Delta \lambda / \lambda^{2}$ ( $c$ est la vitesse de la lumière). Pendant cette durée, la lumière parcourt une longueur $L_{L}$ : la longueur de cohérence longitudinale. C'est la longueur sur laquelle on peut considérer l'onde incidente comme monochromatique. Elle vaut : $L_{L} \equiv \lambda^{2} / \Delta \lambda$. Tant que la différence de chemin optique entre deux faisceaux diffusés est plus petite que $L_{L}$, les franges d'interférences restent visibles. Cette condition de visibilité est moins drastique que la première : elle est en général satisfaite pour un cristal micrométrique, tant que les angles de diffraction ne sont pas trop grands. Sur une source synchrotron, avec un monochromateur classique réglé à $7 \mathrm{keV}$, $L_{L} \sim 0,6 \mu \mathrm{m}$. Ces deux estimations montrent qu'avec une source synchrotron, on peut espérer observer les franges d'interférences d'un objet d'une dizaine de microns. Encore faut-il qu'il reçoive suffisamment de photons..

Une source de rayons X est caractérisée par sa brillance $B$ : c'est le nombre de photons qu'elle émet par seconde, par unité de surface et d'angle solide, dans une bande spectrale (bandwidth ou bw en anglais) relative donnée ${ }^{(1)}$. On l'exprime habituellement en $\mathrm{ph} / \mathrm{s} / \mathrm{mm}^{2} / \mathrm{mrad}^{2} / 0,1 \%$ bw (abrégée en « ub » dans la suite), où $0,1 \%$ bw représente une largeur spectrale relative d'un millième. La brillance est de l'ordre de $10^{20} \mathrm{ub}$ dans les sources synchrotron de $3^{\text {e }}$ génération. Cette grandeur est fondamentale pour l'obtention d'une bonne source cohérente. En effet, on conçoit qu'une source monochromatique de petite taille assure de grandes longueurs de cohérence longitudinale et transverse. Si cette source est puissante et peu divergente, son degré de cohérence sera meilleur. De manière plus quantitative, on montre que le nombre de photons traversant en une seconde une surface limitée par les longueurs de cohérence transverses, le flux cohérent $F_{\text {coh }}$, est donné par l'expression :

$$
F_{c o h}=B \lambda^{2}(\Delta \lambda / \lambda) \times 10^{-8},
$$

où la brillance est exprimée en ub, la longueur d'onde en ångströms et la largeur spectrale relative en millièmes. Pour l'onduleur de la ligne CRISTAL à SOLEIL, où le flux total à $7 \mathrm{keV}$ après un monochromateur $\operatorname{Si}(111)$ est de $6 \times 10^{13} \mathrm{ph} / \mathrm{s}$, le 
>>

flux cohérentvaut $F_{\text {coh }}=2,2 \times 10^{11} \mathrm{ph} / \mathrm{s}$, soit $3 \%$ du flux total! Ainsi, même en utilisant le rayonnement synchrotron, le degré de cohérence est faible. Ceci est encore plus frappant avec les sources de laboratoire. Les meilleures sources actuelles, fonctionnant avec des tubes ou des anodes tournantes, ont des brillances qui atteignent au maximum $10^{11} \mathrm{ub}$, ce qui donne des flux cohérents de l'ordre de $300 \mathrm{ph} / \mathrm{s}$ pour $\lambda=1,54 \AA$, beaucoup trop faibles pour être utilisables.

La brillance est une propriété de la source : elle se conserve si l'optique de la ligne est sans défaut (c'est une conséquence du théorème de Liouville). On ne peut donc augmenter optiquement le flux cohérent. En revanche, il est possible d'augmenter le degré de cohérence en sélectionnant une partie cohérente du faisceau, mais c'est au prix d'une importante perte de flux. En pratique, on insère des paires de fentes près de l'échantillon, que l'on ferme à des tailles voisines des longueurs de cohérence transverses après le passage des optiques (monochromateurs, dispositifs focalisants et filtres). De cette manière, il est possible d'obtenir des flux cohérents compris entre $10^{9}$ et $10^{10} \mathrm{ph} / \mathrm{s}$ dans $10 \times 10 \mu \mathrm{m}^{2}$, ce qui est en général suffisant pour réaliser des expériences de diffraction.

\section{Diffraction cohérente des rayons $X$}

Lorsque l'on place un objet dans un faisceau de rayons $\mathrm{X}$, on observe un diagramme de diffraction qui dépend très fortement de sa structure. Si l'objet est cristallin, le faisceau sera principalement diffracté dans des directions précises, donnant naissance aux taches de Bragg. La cristallographie classique fournit alors des méthodes d'analyse puissantes permettant d'obtenir la position des atomes et même la densité électronique entre et autour de ces atomes. Ces informations sont obtenues dans la "maille ", la boîte à atomes invisible qui se répète périodiquement pour former le cristal. Si l'objet n'est pas cristallin, comme une macromolécule biologique, son diagramme de diffraction est limité aux petits angles de diffraction et les méthodes d'analyse actuelles ne permettent d'obtenir au mieux que sa forme, sans pouvoir déterminer sa structure interne.

Cependant, comme le montrent les figures $2 a-b-c$, les taches de Bragg de cristaux de taille $a$ sont en fait flanquées de franges d'interférence dont la largeur angulaire est de l'ordre de $\lambda / a$. Celles-ci n'apparaissent donc que sous illumination cohérente, comme nous l'avons vu précédemment. Si ces cristaux sont désordonnés, la diffusion due à ce désordre (que l'on nomme "diffusion diffuse ") révèle sous faisceau cohérent une structure fine de tavelures (figs. 2d-e) ou speckles. Quant aux objets non cristallins, comme des macromolécules biologiques, leurs diagrammes de diffraction consistent également en des tavelures, principalement situées aux petits angles. L'utilisation de faisceaux cohérents permet de mesurer ces franges ou ces tavelures, invisibles dans les expériences classiques.

Une des propriétés, remarquable et presque révolutionnaire, de la diffraction cohérente est que les diagrammes obtenus permettent de recouvrer la phase de l'onde diffractée (voir encadré). On peut en effet montrer que si le diagramme de diffraction est connu avec suffisamment de détails pour y séparer les tavelures, et si on connaît avec une bonne approximation la taille de l'objet, il est possible de retrouver la phase de l'onde diffractée en utilisant des algorithmes de "recouvrement de phase». Ce nouveau type d'imagerie, appelé "imagerie par diffraction cohérente" (CDI : Coherent Diffractive Imaging), permet d'obtenir la densité électronique d'un objet avec une résolution qui dépend de l'extension angulaire de l'intensité diffractée mesurable, en pratique quelques dizaines de nanomètres. Les algorithmes de recouvrement de phase sont connus depuis quelques dizaines d'années en optique, mais sont assez récents d'utilisation dans le domaine des rayons $\mathrm{X}$, pour des raisons... de manque de cohérence.

La CDI est une technique exigeante, qui demande un faisceau cohérent intense et une bonne stabilité du montage expérimental. De plus, le fait que la séparation entre les speckles soit faible (avec $\lambda=0,18 \mathrm{~nm}$ et $a=2 \mu \mathrm{m}, \lambda / a \sim 10^{-4}$, ce qui correspond à un écart de $100 \mu \mathrm{m}$ à $1 \mathrm{~m}$ ) exige des détecteurs ayant une résolution suffisamment bonne pour les résoudre. En pratique, on utilise des détecteurs à faible bruit comme les capteurs CCD, dont les pixels ont des tailles de quelques dizaines de microns, mais dont la dynamique est faible, ou de nouveaux détecteurs à pixels hybrides, de meilleure dynamique mais de taille de pixel plus grande $(55,130$ et $170 \mu \mathrm{m})$. Le détecteur de diffraction cohérente parfait reste à inventer !

\section{Diffraction des rayons $X$ et problème des phases}

Lorsqu'on effectue une expérience de diffraction, un faisceau de rayons $\mathrm{X}$ de vecteur d'onde incident $\boldsymbol{k}_{\boldsymbol{l}}$ et de pulsation $\omega$ est dirigé sur un échantillon. Le champ électrique de cette onde incidente, supposée plane (donc cohérente), s'écrit alors $E_{0} \cos \left(\boldsymbol{k}_{l} \cdot r-\omega t\right)$. Ce faisceau est diffusé par un atome $j$ du cristal, avec une amplitude proportionnelle au nombre d'électrons de l'atome. En champ lointain, c'est-à-dire en un point $r$ suffisamment éloigné du cristal pour que l'onde diffusée soit plane (de vecteur d'onde $k_{F}$ ), le champ de l'onde diffusée vaut $E_{j} \cos \left(k_{F} \cdot r-\omega t+\phi_{j}\right)$. La phase de cette onde dépend de la position $r_{j}$ de l'atome et du vecteur de diffusion $q=k_{F}-k_{l}$ par $\phi_{j}=-q . r_{j}$. Le champ total de l'onde diffusée est égal à la somme de tous les champs $: E=\Sigma_{j} E_{j} \cos \left(\boldsymbol{k}_{F} \cdot r-\omega t-q \cdot r_{j}\right)$. L'amplitude de cette onde et sa phase sont donc données par celles du champ complexe $E=\Sigma_{j} E_{j} \exp \left(-\mathrm{i} q . r_{j}\right)$.

Un calcul plus précis montre que ce champ est en fait donné par l'expression : $E(q) \propto \int_{V} \rho(\boldsymbol{r}) \exp (-\mathrm{i} \boldsymbol{q} \cdot \boldsymbol{r}) \mathrm{d}^{3} \boldsymbol{r}$,

où $\rho(r)$ est la densité électronique de l'objet et $V$ le volume de l'échantillon. On reconnaît l'expression d'une transformée de Fourier, qui peut être inversée à condition de connaître la phase de l'onde diffusée. Malheureusement, comme on ne mesure que l'intensité diffractée, proportionnelle à $|E|^{2}$, on ne peut obtenir que son amplitude $|E|$. C'est le problème des phases, bien connu des cristallographes ; $C^{\prime}$ est cette limitation qui est levée par la technique de diffraction cohérente.

Pour un cristal périodique, on peut montrer que le champ $E$ n'est maximum que si le vecteur de diffusion est voisin d'un vecteur $Q_{h k l}$ repéré par trois indices de Miller $h, k$ et $l$, qui indexent le " réseau réciproque » du cristal. Les taches de Bragg correspondantes sont alors repérées par ces trois indices et sont interprétées comme des réflexions sur les plans réticulaires $(h, k, l)$, orthogonaux à $Q_{h k l}$. 

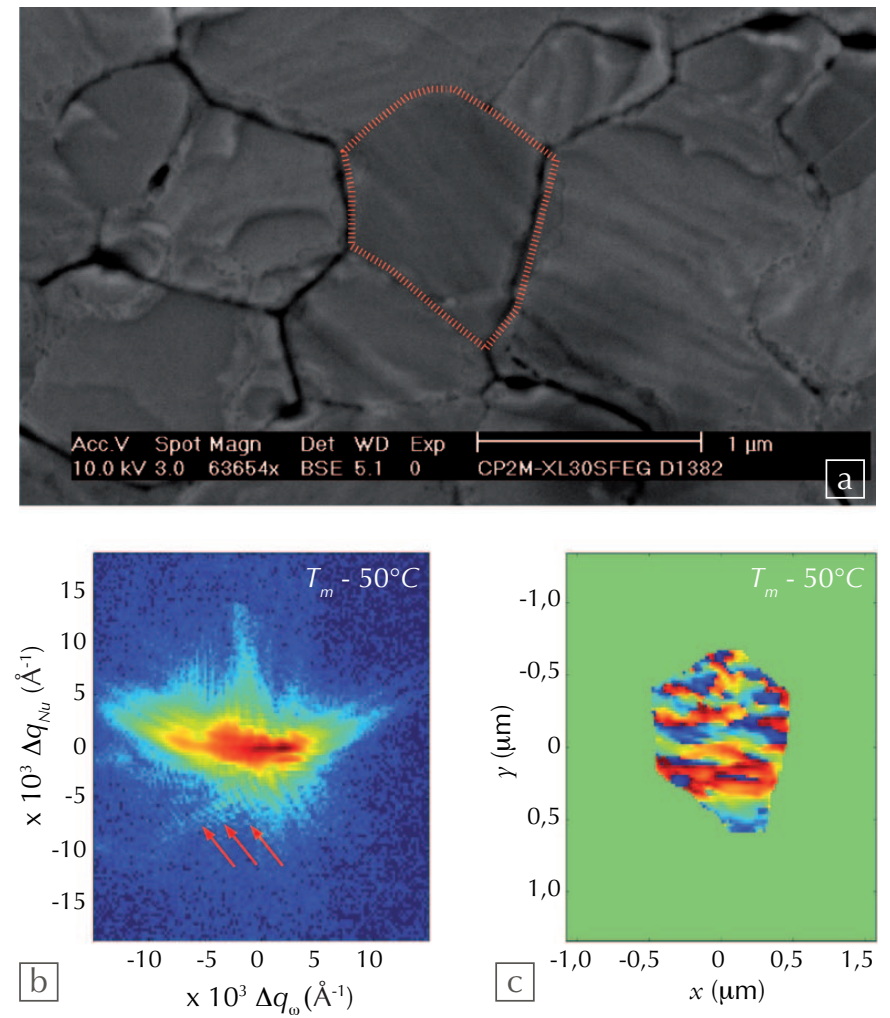

3. Détermination du champ de déformation d'un grain d'or

(a) Image par microscopie électronique à balayage d'un film mince polycristallin d'or, montrant les grains monocristallins de dimensions de l'ordre du micromètre. Le grain étudié est entouré de rouge.

(b) Diagramme de diffraction cohérente de la réflexion (111) du grain montré en (a) : quelques franges d'interférence sont indiquées par les flèches rouges.

(c) Coupe du même grain montrant les champs de déformation obtenus (l'échelle des couleurs, du rouge au bleu, indique que le cristal est plus ou moins déformé).
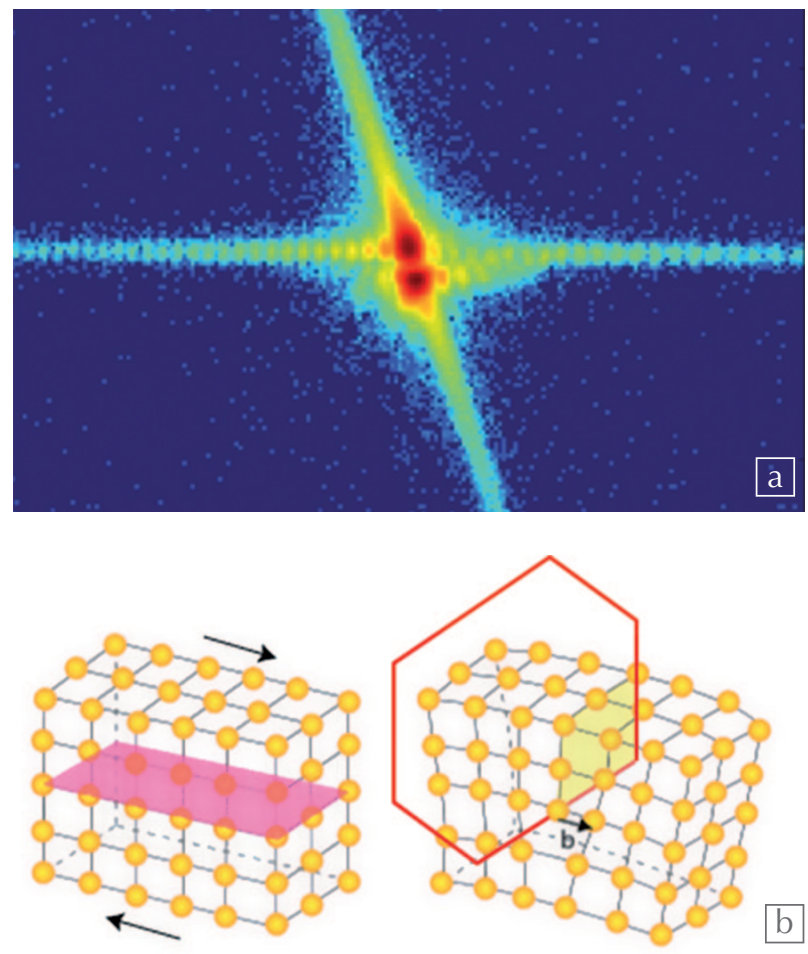

4. Observation d'une dislocation dissociée

(a) Image de diffraction de la réflexion (220) du silicium, lorsque le faisceau de rayons $X$ illumine une dislocation.

(b) Représentation simplifiée d'une dislocation dans un cristal (d'après Wikipédia), créée par un cisaillement élémentaire (image de gauche) ; la ligne rouge sur l'image de droite représente le lieu où le nombre de voisins d'un atome change : c'est la ligne de dislocation. Cette ligne peut faire un circuit fermé, comme indiqué sur le schéma, et former une boucle de dislocation.

\section{Exemple 1 : Reconstruction de la forme et des champs de déplacement d'un grain d'or}

Dans un matériau, les propriétés mécaniques, optiques ou électroniques dépendent fortement des champs de déformations aux échelles nanométriques, c'est-à-dire des variations des positions des atomes par rapport à leurs positions théoriques. Ces déformations peuvent être dues aux défauts cristallins ou aux contraintes mécaniques appliquées au cristal. Elles sont malheureusement très difficiles à mesurer, en particulier dans un matériau fonctionnel. En effet, la technique la plus puissante pour les étudier, la microscopie électronique, est limitée par la faible pénétration des électrons dans la matière, qui rend difficile les études in situ.

Ici, un film d'or de quelques centaines de nanomètres d'épaisseur a été utilisé.
La difficulté principale de l'expérience consistait à faire diffracter un seul grain de ce film polycristallin (fig. 3a), afin de pouvoir reconstruire sa densité et son champ de déplacements. Une fois mesurée la tache de diffraction d'un grain sélectionné (fig. 3b), les algorithmes de recouvrement de phase permettent, connaissant la forme approximative du grain, de déterminer le champ de déplacements avec une résolution de l'ordre de $20 \times 20 \times 20 \mathrm{~nm}^{3}$ (fig. 3c).

Notons que ces résultats originaux ont été obtenus après un travail de longue haleine, en croisant les résultats de diverses techniques de mesures (afin de repérer le grain diffractant) et après optimisation des algorithmes de reconstruction. L'étude a été faite en température et a permis d'aborder la relation entre les propriétés thermomécaniques du film et les déformations locales du grain.

\section{Exemple 2 : Observation de défauts topologiques dans le silicium}

Lorsque certains défauts de la structure sont présents, les champs de déformations varient fortement et de manière discontinue. Ces défauts, comme les dislocations, sont fondamentaux pour expliquer les déformations plastiques des matériaux que nous utilisons quotidiennement. Les dislocations sont bien connues grâce à la microscopie électronique, qui permet de les observer avec des résolutions atomiques, mais avec les mêmes limitations que dans l'exemple précédent.

La figure 4a montre l'image de diffraction de la réflexion (220) d'un cristal de silicium, recuit sous oxygène de manière à obtenir des boucles de dislocations (fig. 4b) d'environ $100 \mu \mathrm{m}$ de diamètre. Le faisceau cohérent, de $10 \times 10 \mu \mathrm{m}^{2}$, a été amené avec soin sur une ligne de 


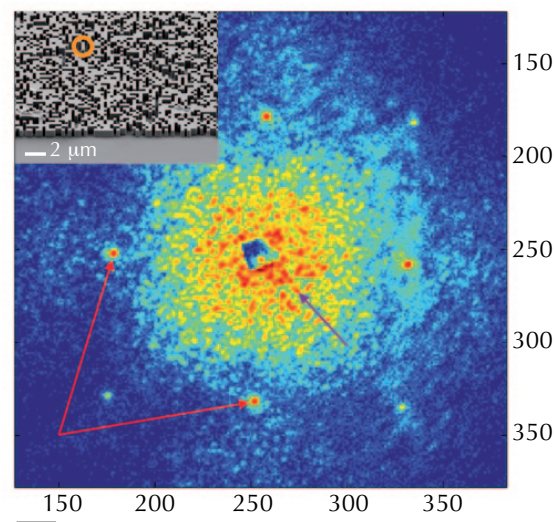

$\mathrm{a}$

\〉

dislocation, ce qui a pour effet de diviser cette tache de diffraction en deux, de manière similaire à l'exemple de la figure $2 \mathrm{f}$. Des effets additionnels sont observés : dans le spectre de diffraction, la ligne horizontale est due au faisceau incident. La ligne continue oblique est due à la dissociation de la ligne de dislocation : dans le silicium, les dislocations se divisent en deux partielles, séparées par une faute d'empilement. Cette première observation d'une dislocation par diffraction cohérente permet des études in situ, non destructives, sous des environnements nouveaux pour l'étude des dislocations, comme par exemple la pression.

\section{Exemple 3 : Ptychographie}

Dans beaucoup de situations, les échantillons étudiés sont plus grands que la taille du faisceau cohérent utilisé. Dans ce cas, les algorithmes de CDI ne fonctionnent plus, parce que la section $\mathrm{du}$ faisceau incident n'est pas aussi clairement définie que la forme de l'objet diffractant. Une autre technique, bien plus efficace, consiste alors à mesurer le diagramme de diffraction pour différentes positions du faisceau sur l'échantillon, à condition que ces positions se recouvrent. Cette technique récente, dite "ptychographie ", permet de faire l'imagerie tridimensionnelle d'un objet quelconque. Elle est de plus en plus utilisée pour imager de petits objets en volume, lorsque les autres techniques sont inefficaces.

En guise d'exemple, la figure 5a montre le diagramme de diffraction d'un objet test, une structure artificielle faite de plots d'or de $800 \mathrm{~nm}$ d'épaisseur et

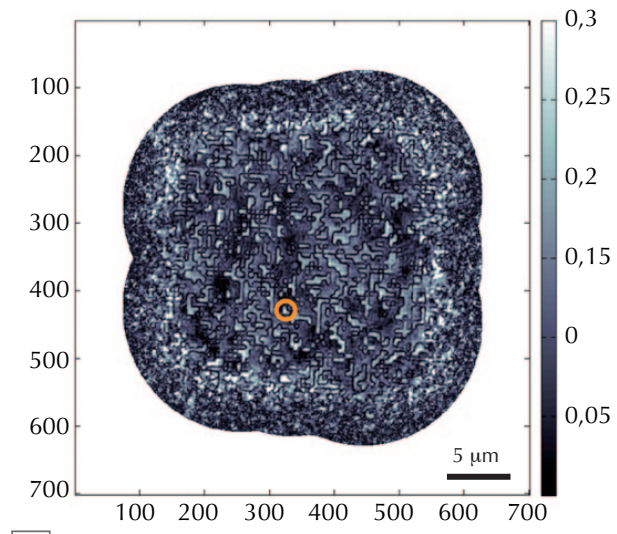

b

\section{Reconstruction ptychographique d'une} structure

(a) Image de diffraction d'une structure artificielle composée de plots d'or. L'encart montre une vue au microscope électronique, où la surface de ces plots apparaît en gris clair (le cercle orange montre trois plots adjacents formant un \langle|$»)$. Les tavelures de centre du diagramme sont clairement visibles (flèche violette), ainsi que les taches de Bragg (flèches rouges).

(b) Reconstruction ptychographique de la structure, à partir du diagramme de diffraction. On reconnaît les plots d'or au centre de l'image. Le cercle orange entoure trois plots adjacents formant un « $L »$. de $350 \mathrm{~nm}$ de côté (voir insert), disposés aléatoirement sur un réseau. Le diagramme de diffraction mesuré pour une position donnée du faisceau montre des pics de Bragg fins dus au réseau de plots, et des tavelures dues à leur désordre. De telles images sont collectées en fonction de la position du faisceau, qui décrit ici une trajectoire en spirale sur l'échantillon. La reconstruction ptychographique n'est effective que dans la zone centrale de la figure $5 b$, où il y a recouvrement des diverses positions du faisceau. Les plots d'or y sont visibles.

\section{Conclusion}

La diffraction cohérente est apparue dans les années 1990 dans les centres de rayonnement synchrotron, en particulier à l'ESRF. La cohérence des rayons X étant une propriété pour l'instant impossible à obtenir de manière utilisable dans les laboratoires, cette technique de diffraction s'est principalement développée autour des grands instruments, comme le synchrotron SOLEIL. Elle ne peut que continuer à le faire, car toutes les nouvelles sources en construction dans le monde ont ou auront des propriétés de cohérence encore meilleures. C'est le cas par exemple du premier laser à électrons libres X, le Linear Coherent Light Source (LCLS) de Stanford, entré en fonctionnement en avril 2009.

De par les possibilités nouvelles qu'elle offre, la diffraction cohérente de rayons $\mathrm{X}$ devient une technique de plus en plus importante dans la panoplie des chercheurs en matière condensée. I
(1) La brillance est similaire à la "luminance spectrique ", qui est une grandeur normalisée, utilisée en optique. La brillance n'est pas une grandeur normalisée, mais elle est très utilisée dans le domaine des sources de rayonnement synchrotron. C'est pourquoi nous l'avons conservée dans cet article, ainsi que son unité.

\section{Remerciement}

Les exemples exposés dans cet article ont été obtenus sur la ligne CRISTAL du synchrotron SOLEIL (grain d'or, dislocation et ptychographie) et la ligne ID01 de l'ESRF (grain d'or). L'étude du grain d'or fait partie de la thèse de Nicolas Vaxelaire, de l'Institut des matériaux, de microélectronique et des nanosciences de Provence, et a été réalisée en collaboration avec C. Kirchlechner, J. Keckes, Erich Schmid, Stéphane Labat et Olivier Thomas. Les collaborateurs sur la ligne ID01 étaient T. Cornelius, T. Schulli, A. Diaz et T. Metzger. La dislocation dans le silicium a été étudiée dans le travail de thèse de Vincent Jacques, maintenant au Laboratoire de Physique des solides d'Orsay, en collaboration avec David Le Bolloc'h, Frédéric Livet, Guy Rolland et Michèle Sauvage. La ptychographie a été réalisée par Felisa Berenguer, alors au London Centre for Nanotechnology, avec ses collaborateurs Laura Shemilt, Richard Bean, Laurent Bozec et Ian K. Robinson.

\section{En savoir plus}

- F. Livet, "Diffraction with a coherent X-ray beam dynamics and imaging", Acta Cryst. A63 (2007) 87-107.

- N. Vaxelaire et al., New Journal of Physics 12 (2010) 035018.

- V. Jacques et al., Phys. Rev. Lett. 106 (2011) 065502.

- F. Berenguer de la Cuesta et al., "Coherent diffraction from collagenous soft tissues", PNAS, 106, (2009) 15297. 